\title{
Increased cyclic guanosine monophosphate levels and continuous-flow left-ventricular assist devices: Implications for gastrointestinal bleeding
}

\author{
Liza Grosman-Rimon, PhD, ${ }^{\mathrm{a}, \mathrm{b}}$ Laura C. Tumiati, BSc, ${ }^{\mathrm{a}}$ Avi Fuks, MD, ${ }^{\mathrm{a}}$ Ira Jacobs, DrMedSci, ${ }^{\mathrm{b}}$ \\ Spencer D. Lalonde, HBSc, ${ }^{\mathrm{a}}$ David Z. I. Cherney, $\mathrm{MD}, \mathrm{PhD},{ }^{\mathrm{c}}$ and Vivek Rao, $\mathrm{MD}, \mathrm{PhD}^{\mathrm{a}}$
}

\begin{abstract}
Objectives: We examine the hypothesis that cyclic guanosine monophosphate (cGMP) levels are elevated in recipients of continuous-flow left ventricular assist devices (CF-LVADs) and that elevated cGMP levels are associated with a risk of gastrointestinal (GI) bleeding events.

Methods: The levels of cGMP, nitric oxide, platelet activation markers, plateletderived growth factors (PDGF) $\mathrm{AB} / \mathrm{BB}$ and $\mathrm{AA}$, and the inflammatory mediator C-reactive protein (CRP) were examined in 19 CF-LVAD recipients, 21 patients who had heart failure, and 19 healthy control-group participants.
\end{abstract}

Results: The median level of cGMP was significantly higher in CF-LVAD recipients, compared with healthy participants $(6.6$ vs $2.1 \mathrm{pmol} / \mathrm{mL}, u=62.5$; $P=.001 ; r=-0.55)$. Median cGMP levels in the heart failure group (12.5 $\mathrm{pmol} / \mathrm{L})$ were higher, compared with both CF-LVAD recipients $(u=75.0$; $P=.001 ; r=-0.53)$ and healthy participants $(u=4.0 ; P<.001 ; r=-0.83)$. Compared with the healthy group, median CRP levels were significantly higher in CF-LVAD recipients $(2.9 \mathrm{vs} 8.0 \mathrm{mg} / \mathrm{L} ; u=58.0 ; P<.001 ; r=-0.63)$ and heart failure patients $(2.9 \mathrm{vs} 7.0 \mathrm{mg} / \mathrm{L} ; u=59.0 ; P<.001 ; r=-0.65)$. In the subgroup of patients supported with the HeartMate II (Thoratec Corporation, Pleasanton, Calif), pulsatility index was significantly negatively correlated with cGMP levels $(r=-0.73 ; P<.05)$, indicating that low pulsatility index is associated with higher cGMP levels. High cGMP levels were significantly associated with GI bleeding events, but not with bleeding events in general.

Conclusions: The primary finding of this study is that GI bleeding in CF-LVAD recipients is associated with significantly elevated cGMP levels, despite high levels of CRP, which interfere with cGMP production. Further studies are required to determine whether elevated cGMP levels can be used as a clinical marker for increased risk of GI bleeding in CF-LVAD recipients. ( $\mathrm{J}$ Thorac Cardiovasc Surg 2016;151:219-27)

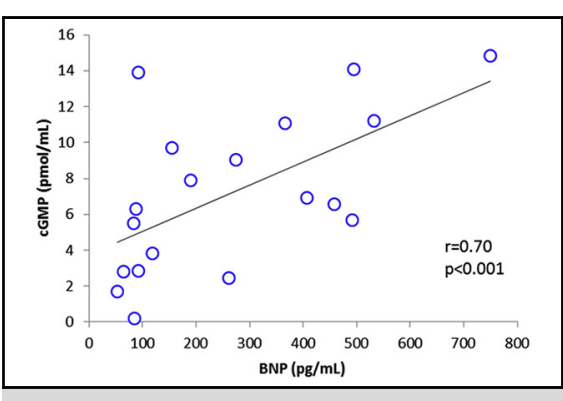

In CF-LVAD recipients, cGMP levels were significantly correlated with the levels of BNP. While elevated cGMP levels predicted Gl Bleeding events, the levels of BNP did not predict bleeding events in these patients.

\section{Central Message}

In recipients of CF-LVAD, GI bleeding events were associated with high cGMP levels.

\section{Perspective}

High cGMP levels may increase the likelihood of GI bleeding events in CF-LVAD recipients, via impaired platelet aggregation. Further studies are required to determine whether elevated cGMP levels can be used as a clinical marker for increased risk of GI bleeding in $\mathrm{CF}$ LVAD recipients.

See Editorial Commentary page 228.

See Editorials page 10 and 13.

\footnotetext{
From the ${ }^{\mathrm{a}}$ Division of Cardiovascular Surgery, Peter Munk Cardiac Centre, University Health Network, Toronto, Canada, 'bepartment of Exercise Sciences, Faculty of Kinesiology and Physical Education, University of Toronto, Toronto, Canada, and 'Division of Nephrology, University Health Network, Toronto, Canada.

D.Z.I.C. and V.R. are co-senior authors.

Received for publication June 8, 2015; revisions received Aug 11, 2015; accepted for publication Sept 4, 2015; available ahead of print Oct 26, 2015.

Address for reprints: Vivek Rao, MD, PhD, Division of Cardiovascular Surgery, Peter Munk Cardiac Centre, University Health Network, University of Toronto, 4N - 457, 200 Elizabeth St, Toronto, Ontario, Canada M5G 2C4 (E-mail: Vivek. Rao@uhn.ca).

$0022-5223 / \$ 36.00$

Copyright (C) 2016 by The American Association for Thoracic Surgery

http://dx.doi.org/10.1016/j.jtcvs.2015.09.015
}

Left ventricular assist devices have been developed as mechanical supports to improve or replace the functions of a failing heart. The newer continuous-flow left ventricular assist devices (CF-LVADs) have been shown to confer advantages, compared with the older pulsatile pumps, including greater durability, smaller size, fewer associated infections, ${ }^{1,2}$ and most notably, increased survival rates. ${ }^{3}$ However, general increases in bleeding, particularly gastrointestinal (GI) bleeding, are a major source of frequent adverse events in patients supported by CF-LVADs. ${ }^{4,5}$ A recent report suggests that, 


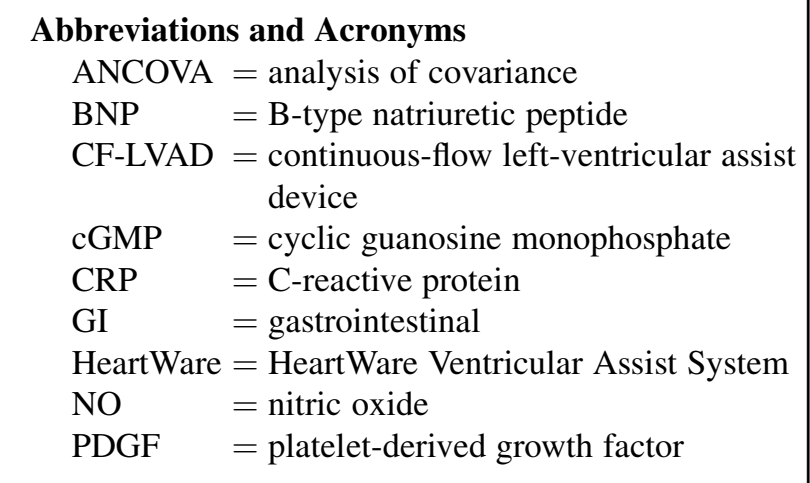

compared with pulsatile pumps, rates of GI bleeding with CF-LVADs are significantly higher. ${ }^{6}$ Given that GI bleeding can be a substantial complication of CF-LVAD therapy, the mechanisms of GI bleeding are important to understand.

In patients with end-stage renal disease, GI bleeding is a well known complication. ${ }^{7-9}$ One study of such patients found that defective platelet aggregation is associated with exaggerated production, by the platelets, of nitric oxide (NO) and of cyclic guanosine monophosphate (cGMP). ${ }^{10}$ In uremic animal models, blocking of NO-cGMP production decreased bleeding time. ${ }^{11}$ This effect on bleeding time was completely reversed by the NO precursor L-arginine, suggesting that elevated cGMP levels, contribute to platelet dysfunction.

The mechanisms leading to GI bleeding in patients with CF-LVAD support may be similar to those responsible for uremic bleeding. Specifically, a higher incidence of GI bleeding in CF-LVAD recipients may be related to augmented NO-cGMP levels, because cGMP impairs platelet activation. ${ }^{12}$ In addition, studies have shown that the contact of blood with an artificial surface activates platelets and causes the $\alpha$-granules to release their contents, which include platelet-derived growth factor (PDGF). ${ }^{13-15}$ In patients supported with CF-LVADs, blood is in contact with the surface of the surgically implanted mechanical pump.

Studies have shown that shortly after CF-LVAD implantation, levels of inflammatory markers are elevated. ${ }^{16,17}$ C-reactive protein (CRP), an important marker of systemic inflammation, is known to decrease NO-cGMP production levels by interfering with endothelial NO synthase expression. ${ }^{18}$ Despite this increase in CRP, which potentially interferes with NO-cGMP production, and the possible activation of platelets by contact of the blood with the surface of the CF-LVAD, bleeding is a common adverse event in this patient population. ${ }^{5}$ Thus, high levels of NO and cGMP may impair platelet activation.
In this study, we examined the hypothesis that cGMP levels are elevated in CF-LVAD recipients and are associated with the risk of GI bleeding events. The specific aims were as follows: (1) to examine the levels of cGMP, NO, PDGF, and CRP in CF-LVAD recipients and compare them to those of heart failure patients, and with healthy controls; and (2) to assess whether high cGMP levels are associated with either a risk of bleeding in general or with GI bleeding in particular in CF-LVAD recipients.

\section{METHODS \\ Clinical Data}

The study protocol was reviewed and approved by the University Health Network Ethical Review Board. Informed consent was obtained from each participant before the study. The total of 59 participants was comprised of 21 heart failure patients, 19 CF-LVAD recipients, and 19 healthy controls, all aged 30-72 years. The CF-LVAD recipients, who were supported for at least 2 months, were compared with heart failure patients, who received standard care, and healthy participants in terms of cGMP $(\mathrm{pmol} / \mathrm{mL}), \mathrm{NO}(\mu \mathrm{mol} / \mathrm{L}), \mathrm{CRP}(\mathrm{mg} /$ L), and PDGF levels.

The mean CF-LVAD treatment time, from implantation, was $9.9 \pm 1.4$ months (range: 2-27.5 months). Eight CF-LVAD recipients were supported by the HeartWare Ventricular Assist System (HeartWare; HeartWare International, Inc, Framingham, Mass); 9 by the HeartMate II (Thoratec Corporation, Pleasanton, Calif); and 2 by the DuraHeart (Terumo Heart, Ann Arbor, Mich). Among the 19 CF-LVAD recipients, 6 (32\%) had general bleeding events, and $4(21 \%)$ had GI bleeding events.

Blood samples were obtained during hospital admission for either GI or general bleeding. In the cohort of patients who did not have evidence of bleeding, blood samples were collected during a routine clinic visit at an average of 9.8 months after CF-LVAD implantation.

A GI bleeding event was defined as: (1) bleeding identified by endoscopy; or (2) heme-positive stool and a decrease in hemoglobin $>1 \mathrm{~g} / \mathrm{dL}$. The international normalized ratio, white blood cell count $\left(\times 10^{9} / \mathrm{L}\right)$, and lactate dehydrogenase (U/L) were recorded. GI bleeding was identified in the upper and lower GI track, requiring intervention, including the temporary cessation of anticoagulation. A general bleeding event was defined as any type of bleeding (with the exclusion of a GI bleeding event). Age and heart failure etiology, the levels of B-type natriuretic peptide (BNP), creatinine, estimated glomerular filtration rate, and medication usage were documented.

\section{Biochemical Assays}

Blood samples were obtained and collected in chilled tubes containing ethylendiaminetetraacetic acid (EDTA). The samples were centrifuged at $2056 \mathrm{~g}$ for 15 minutes at $4{ }^{\circ} \mathrm{C}$, and the plasma was stored at $-80^{\circ} \mathrm{C}$ until analysis. Concentrations of PDGF AA and $\mathrm{AB} / \mathrm{BB}$ were measured in duplicate, using Milliplex human cytokine kit (EMD Millipore, St. Charles, Mo), following manufacturer directions. The patient samples were loaded onto a 96-well plate, together with appropriate standards and controls, and were run in duplicate. Antibodyimmobilized beads were added to the wells. The beads were conjugated to antibodies for the PDGF antigens in the panel. After an incubation period, the unbound beads were removed, and an antibody detection cocktail solution was added to the wells, along with streptavidinphycoerythrin for visualization. The plate was run on a Luminex 200 machine (Luminex Corp, Austin, Tex), and the levels of PDGF were quantified. Plasma CRP was measured by latex-enhanced nephelometry 
on a Siemens BNII nephelometer analyzer (Siemens Healthcare Diagnostics, Germany).

The plasma concentration of total NO was determined in duplicate, using commercially available assay kits (R\&D Systems, Inc, Minneapolis, Minn). Given that NO is an unstable compound that has a very short half-life of seconds in vivo, measuring it directly is difficult. Therefore, the levels of the more stable metabolites nitrite and nitrate were used as an indirect measure of NO. The assay for the determination of total NO in plasma is based on the concentration of nitrite and nitrate, the latter of which is converted to nitrite by the enzyme nitrate reductase.

After this enzymatic process, a colorimetric detection of the nitrite is performed through the Greiss reaction. The presence of organic nitrite compounds is detected by the formation of a red pink color upon treatment of nitrite in the sample with the reagents. Through a 2-step diazotization, acidified nitrite produces a nitrosating agent, which reacts with the coenzyme sulfanilic acid (sulfanilamide and $2 \mathrm{~N}$ hydrochloric acid) to produce the diazonium ion. This ion is coupled with ethylenediamine to form a chromophoric azo-dye derivative that absorbs light at 540 to $570 \mathrm{~nm}$. A microplate reader set at $540 \mathrm{~nm}$, with $690 \mathrm{~nm}$ as the reference, determines the optical density of each well.

Plasma cGMP levels were determined using an enzyme immunoassay kit (Cayman Chemical Co, Ann Arbor, Mich). This assay is based on the competition between free cGMP and the enzyme cGMP-acetylcholinesterase conjugate for cGMP-specific rabbit antibody-binding sites. Given that the concentration of this enzyme conjugate is known, whereas the concentration of cGMP in the plasma varies, the amount of the enzyme conjugate that binds to the rabbit antibody will be inversely proportional to the concentration in the plasma. The binding was maximized by the acetylation of the cGMP prior to loading of the plate. The rabbit antibody-cGMP (free or tracer) complex bound onto the mouse monoclonal antirabbit IgG, which coats the wells.

The plate was washed to remove any unbound reagents; Ellman's Reagent (Caymen Chemical Co, Ann Arbor, Mich) containing the substrate for acetylcholinesterase was added to the wells, resulting in an enzymatic reaction and the formation of a yellow color. The color intensity was determined spectrophotometrically, and was proportional to the amount of enzyme conjugate bound to the well, which was inversely proportional to the amount of free cGMP in the plasma.

\section{Statistical Analysis}

For data sets that were normally distributed, a 1-way analysis of variance was performed with Tukey's post-hoc analysis to examine the differences among CF-LVAD recipients, a healthy control group, and heart failure patients. Data were presented as mean and SD. For data sets that were not normally distributed, the Kruskal-Wallis analysis, followed by the Mann-Whitney $U$ test with Bonferroni correction, was performed to assess the differences. Cohen's effect size estimates were calculated (reported as an $r$ value). Data were presented as a median and interquartile range. An analysis of covariance (ANCOVA) was performed to compare patients who had CF-LVAD, those who had heart failure, and a healthy control group, while holding all medications constant. The medications that may increase cGMP levels were considered as covariates, including sildenafil, hydralazine, aspirin, warfarin, and aspirindipyridamole.

Logistic regression analysis was performed to assess whether cGMP levels are associated with general and GI bleeding events, with use of anticoagulants as a covariate. Spearman's rank correlation coefficient was performed to assess the relationship between the biomarkers. Cox regression analysis was performed to examine the association of cGMP levels and anticoagulants as covariates with hazards of GI or general bleeding events. The Fisher exact test was performed to compare differences in cGMP levels for the following: patients with GI versus without GI bleeding events; patients with general versus without general bleeding events; patients with a closed versus open aortic valve; and patients with ischemic versus nonischemic heart failure etiology. A $P$ value $\leq .05$ was considered significant.

\section{RESULTS}

\section{Participant Characteristics}

In patients with CF-LVAD support, the median BNP level was significantly lower than that of heart failure patients (172.7 vs $625.0 \mathrm{pg} / \mathrm{mL} ; u=82.5 ; P=.03, r=-0.48$ ), but significantly higher than that of healthy participants $(13.4 \mathrm{pg} / \mathrm{mL} ; u=0.0 ; P<.001 ; r=-0.84$ ) (Figure $1, A$ ). The estimated glomerular filtration rate was not significantly different in patients who had heart failure, compared with CF-LVAD recipients, whereas creatinine level was significantly higher (Table 1). None of the study participants required dialysis. The percentage of patients with ischemic etiology was similar in the group of patients with heart failure $(38 \%)$ and the group who received a CF-LVAD $(37 \%)$.

\section{Data for CF-LVAD Recipients}

The mean time to the first GI bleeding event from the time of CF-LVAD implantation was 8.3 months (range: 5-12 months), whereas the mean time to first general bleeding event from the time of CF-LVAD implantation was 10.5 months (range: 3.0-27.5 months). The median international normalized ratio was 2.05 (interquartile range: 1.76-2.23); and lactate dehydrogenase (U/L) was 265 (interquartile range: 265-471). The mean white blood cell count was $6.3 \pm 1.6\left(\times 10^{9} / \mathrm{L}\right)$. As confirmed by echocardiography, the aortic valve was closed in about $63 \%$ of the CF-LVAD recipients, and open every cycle or intermittently in only $37 \%$ of the recipients.

\section{Plasma Levels of Biomarkers}

The median level of cGMP was significantly higher in CF-LVAD recipients, compared with healthy controls (6.6 vs $2.1 \mathrm{pmol} / \mathrm{mL} ; u=62.5 ; P=.001 ; r=-0.55)$. However, median cGMP levels in the group who had heart failure $(12.5 \mathrm{pmol} / \mathrm{L})$ were higher, compared with those of both CF-LVAD recipients $(u=75.0 ; P=.001 ; r=-0.53)$ and healthy participants $(u=4.0 ; P<.001 ; r=-0.83)$ (Figure 1, B). The median PDGF AA levels were significantly higher in the healthy participants, compared with patients who had heart failure $(P<.05)$, whereas differences between CF-LVAD recipients and other groups did not reach significance (Table 2). No significant differences in the levels of PDGF $\mathrm{AB} / \mathrm{BB}$ and $\mathrm{NO}$ were found among the groups (Table 2).

Compared with those of the healthy participants, median CRP levels were significantly higher in both CF-LVAD 


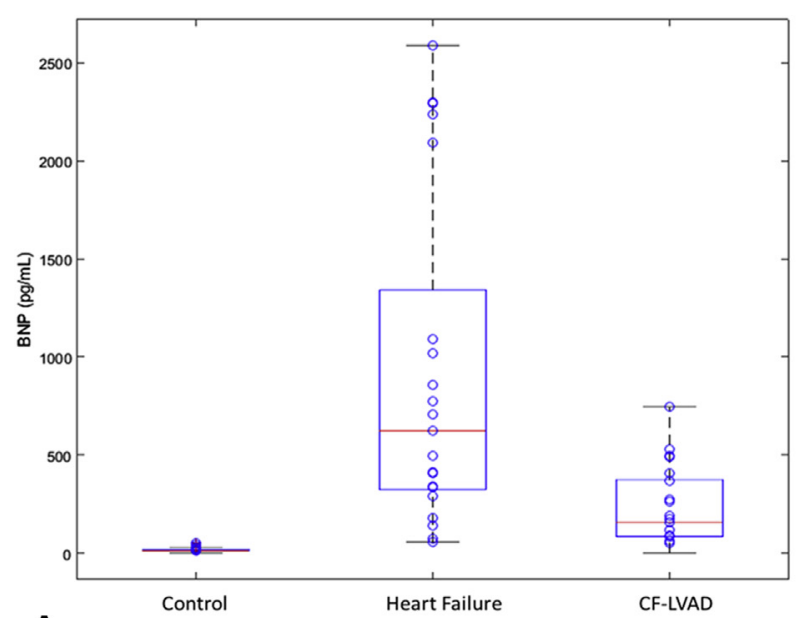

A

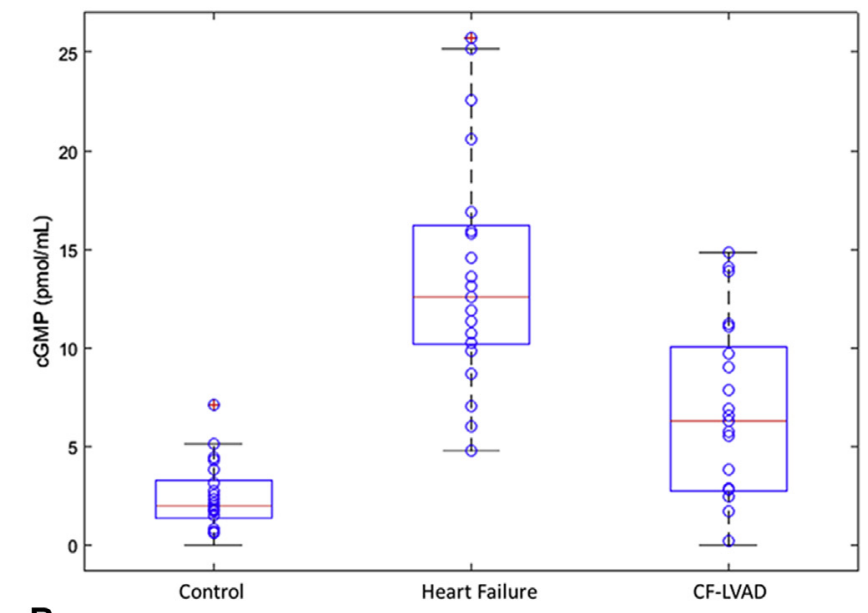

B

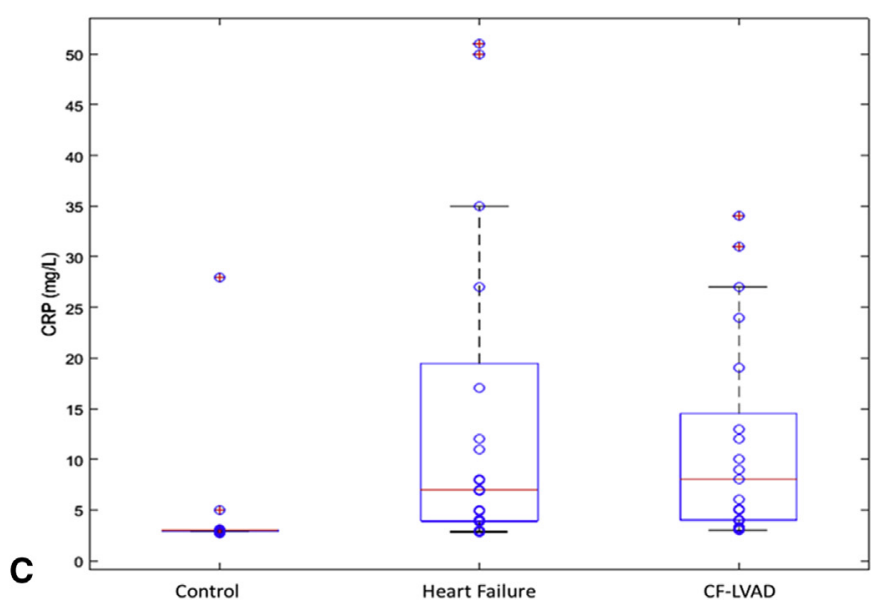

FIGURE 1. The top and bottom borders of the box mark the 75th and 25th percentiles, respectively; whiskers mark the 90th and 10th percentiles. A circle with a cross represents extreme outliers beyond the 90th and 10th percentiles; horizontal red line indicates the median. In patients with heart failure, CF-LVAD recipients, and healthy controls: (A) levels of BNP (pg/mL); (B) levels of cGMP (pmol/mL); and (C) levels of CRP (mg/L). BNP, B-type natriuretic peptide; $C F-L V A D$, continuous flow left-ventricular assist device; $c G M P$, cyclic guanosine monophosphate; $C R P$, C-reactive protein.

recipients $(2.9$ vs $8.0 \mathrm{mg} / \mathrm{L} ; u=58.0 ; P<.001 ; r=-0.63)$ and patients who had heart failure $(2.9$ vs $7.0 \mathrm{mg} / \mathrm{L}$; $u=59.0 ; P<.001 ; r=-0.65)$, whereas no significant differences were observed between CF-LVAD recipients and patients who had heart failure (Figure 1,C). Despite the high CRP levels in the latter 2 groups, cGMP levels were significantly higher in these 2 groups, compared with the healthy participants (Figure 1,C).

An ANCOVA was performed to examine the differences in cGMP levels among the groups, while holding the effects of the medications constant. Sildenafil, hydralazine, aspirin, warfarin and aspirin-dipyridamole were entered into the model as covariates. The ANCOVA revealed that only sildenafil affects cGMP levels $(P<.01)$. Other covariates, including these drugs, did not contribute significantly to the levels of cGMP. When the effects of these drugs were removed by performing the ANCOVA, the CF-LVAD recipients continued to have significantly higher cGMP levels than did the healthy control group $(P<.01)$. In addition, in patients with CF-LVAD support, sildenafil use alone was not significantly associated with cGMP levels. In CF-LVAD recipients who had general bleeding events $(\mathrm{n}=6), 1$ used aspirin-dipyridamole, 5 used warfarin and 5 aspirin; in those who had GI bleeding $(n=4), 1$ used aspirin-dipyridamole, 3 warfarin, 2 aspirin, 1 sildenafil, and 1 hydralazine.

In CF-LVAD recipients, cGMP levels were higher in those who had GI bleeding events, versus those who had no GI bleeding events (Table 3). No significant differences were observed in cGMP levels between CF-LVAD recipients who had general bleeding events, compared with those with no general bleeding events (Table 3). The levels of cGMP were not significantly different for CF-LVAD recipients with ischemic versus nonischemic etiology of heart failure. 
TABLE 1. Clinical data for patients with heart failure versus those who received CF-LVADs

\begin{tabular}{|c|c|c|}
\hline Clinical data & $\begin{array}{l}\text { Patients with } \\
\text { heart failure } \\
\quad(n=21)\end{array}$ & $\begin{array}{c}\text { Patients who } \\
\text { received CF-LVADs } \\
(n=19)\end{array}$ \\
\hline \multicolumn{3}{|l|}{ CF-LVAD } \\
\hline Length of support (mo) & - & $9.9 \pm 5.9$ \\
\hline Flow (L/min) & - & $4.4 \pm 0.6$ \\
\hline Motor power (watt) & - & $4.1 \pm 1.8$ \\
\hline Pulsatility index & - & $5.7 \pm 0.5$ \\
\hline Speed (RPM) & - & $5163.3 \pm 3227.9$ \\
\hline \multicolumn{3}{|l|}{ Heart } \\
\hline Aortic valve opening & - & $7(36.8)$ \\
\hline Ischemic etiology & $8(38.0)$ & $7(36.8)$ \\
\hline \multicolumn{3}{|l|}{ Kidney } \\
\hline eGFR $\left(\mathrm{mL} / \mathrm{min} / 1.73 \mathrm{~m}^{2}\right)^{*}$ & $54.9 \pm 27.6$ & $67.9 \pm 24.5$ \\
\hline Creatinine $(\mu \mathrm{mol} / \mathrm{L}) \dagger$ & $134.5 \pm 56.2$ & $97.7 \pm 31.6$ \\
\hline \multicolumn{3}{|l|}{ Drugs (mg; n [\%]) } \\
\hline Sildenafil (60-120) & $2(2.5)$ & $4(21)$ \\
\hline Hydralazine (30-125) & $3(14.2)$ & $4(21)$ \\
\hline Aspirin (81-325) & $14(73.6)$ & $12(57.1)$ \\
\hline Warfarin (2-6.25) & $15(71.4)$ & $17(89.4)$ \\
\hline Aspirin-dipyridamole (25) & - & $1(5.2)$ \\
\hline Digoxin & $7(33.3)$ & $5(26.3)$ \\
\hline Furosemide & $18(85.7)$ & $14(73.6)$ \\
\hline Beta blockers & $15(71.4)$ & $11(57.8)$ \\
\hline ACE inhibitors & $9(42.8)$ & $11(57.8)$ \\
\hline Angiotensin receptor blockers & $5(23.8)$ & $1(5.2)$ \\
\hline Aldosterone antagonists & $12(57.1)$ & $15(78.9)$ \\
\hline Proton pump inhibitor & $3(14.2)$ & $11(57.8)$ \\
\hline Statins & $33.3(7)$ & $31.5(6)$ \\
\hline Antiarrhythmic drug & $8(38)$ & $7(36.8)$ \\
\hline
\end{tabular}

In our study, GI bleeding in the CF-LVAD recipients was not associated with their age; and age was not associated with cGMP, NO, or PDGF AA or AB/BB levels. No associations were found between length of the CF-LVAD support and cGMP, or PDGF $\mathrm{AA}$ or $\mathrm{AB} / \mathrm{BB}$ levels. However, length of the support was positively correlated with levels of NO (Spearman's rho $=0.62 ; P<.01$ ) (Figure 2, $A$ ).

In CF-LVAD recipients, the levels of cGMP were significantly correlated with BNP levels (Spearman's rho $=0.70 ; P=.001$ ) (Figure 2, B). No significant correlation was observed between levels of cGMP and BNP for either the group with heart failure or the healthy control group. The levels of cGMP were moderately negatively correlated with the levels of both PDGF AA (Spearman's rho $=-0.51 ; P=.025)$ and $\mathrm{PDGF} \mathrm{AB} / \mathrm{BB}$ (Spearman's rho $=-0.53 ; P=.02$ ) in the group who received a CF-LVAD, but not in the group who had heart failure. However, after controlling for aspirin, aspirin-dipyridamole, and sildenafil, neither PDGF AA nor $A B / B B$ levels were significantly correlated with cGMP levels. In the CF-LVAD recipients, the levels of cGMP were not significantly correlated with CRP, lactate dehydrogenase, or white blood cell levels. In addition, no significant relationships were found between cGMP levels and either estimated glomerular filtration rate or creatinine levels. Pulsatility index was only available for the HeartMate II devices $(n=9)$. For those patients, pulsatility index was significantly negatively correlated with cGMP levels (Spearman's rho $=-0.73 ; P<.05$ ), indicating that a low pulsatility index is associated with higher cGMP levels.

\section{General and GI Bleeding Events}

Logistic regression indicated that cGMP levels are significantly associated with GI bleeding, but not with bleeding in general. In CF-LVAD recipients, the regression coefficient (B) indicated that for every unit increase in cGMP (pg/mL), the odds ratio of GI bleeding versus not bleeding increases by $1.48(P<.05)$. However, the levels of cGMP did not predict overall bleeding events.

The levels of NO and BNP did not significantly predict bleeding in general or GI bleeding events. Neither lactate

TABLE 2. Recipients of a CF-LVAD compared with healthy control-group participants

\begin{tabular}{|c|c|c|c|c|}
\hline Characteristics & Healthy control $(n=19)$ & Heart failure $(n=21)$ & CF-LVAD $(n=19)$ & $P$ value \\
\hline General bleeding, $\mathrm{n}(\%)$ & $0(0)$ & $0(0)$ & $31.5(6)$ & - \\
\hline GI bleeding, $\mathrm{n}(\%)$ & $0(0)$ & $0(0)$ & $21(4)$ & - \\
\hline Gender, male, n (\%) & $12(63.1)$ & $15(71.4)$ & $10(52.6)$ & 一 \\
\hline Height $(\mathrm{cm})$ & $168.5 \pm 10.8$ & $172.2 \pm 11.9$ & $170.3 \pm 13.1$ & .62 \\
\hline Weight $(\mathrm{kg})$ & $73.9 \pm 13.1$ & $82.9 \pm 20.7$ & $74.0 \pm 14.9$ & .15 \\
\hline Body mass index $\left(\mathrm{kg} / \mathrm{m}^{2}\right)$ & $26.0 \pm 4.2$ & $27.7 \pm 6.0$ & $25.3 \pm 2.9$ & .24 \\
\hline $\mathrm{NO}(\mu \mathrm{moL})$ & $44.8(38.6,94.7)$ & $48.3(40.6,71.9)$ & $78.3(45.8,98.0)$ & .07 \\
\hline PDGF AB/BB (pg/mL) & $824.8(275.0,1258.9)$ & $180.3(116.5,508.7)$ & $134.7(85.1,599.1)$ & .09 \\
\hline PDGF AA $(\mathrm{pg} / \mathrm{mL})$ & $15.5(7.7,32.7)$ & $6.2(3.5,13.1)$ & $9.5(3.9,19.1)$ & $.05^{*}$ \\
\hline
\end{tabular}

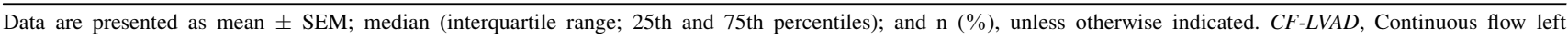
ventricular assist devices; $G I$, gastrointestinal; $N O$, nitric oxide; $P D G F$, platelet-derived growth factor. * Significant differences between heart failure and health control-group subjects. 
TABLE 3. Levels of biomarkers in the CF-LVAD recipient group

\begin{tabular}{lcccccc}
\hline \multicolumn{1}{c}{ Biomarkers } & $\begin{array}{c}\text { No general bleeding } \\
\mathbf{n}=\mathbf{1 3}\end{array}$ & $\begin{array}{c}\text { General bleeding } \\
\mathbf{n = 6}\end{array}$ & $\boldsymbol{P}$ value & $\begin{array}{c}\text { No GI bleeding } \\
\mathbf{n}=\mathbf{1 5}\end{array}$ & $\begin{array}{c}\text { GI bleeding } \\
\mathbf{n}=\mathbf{4}\end{array}$ & $\boldsymbol{P}$ value \\
\hline BNP $(\mathrm{pg} / \mathrm{mL})$ & $105.8(84.2,343.5)$ & $340.6(137.5,503.8)$ & .21 & $105.8(84.5,376.7)$ & $384.1(184.8,685.4)$ & .07 \\
cGMP $(\mathrm{pmol} / \mathrm{mL})$ & $6.3(2.8,10.0)$ & $7.8(2.9,10.4)$ & .77 & $5.7(2.8,7.9)$ & $11.9(9.2,14.7)$ & .01 \\
NO $(\mu \mathrm{moL})$ & $81.5(57.0,103.5)$ & $71.2(45.1,137.6)$ & .83 & $84.5(46.3,105.6)$ & $64.7(46.0,108.2)$ & .73 \\
PDGF AB/BB $(\mathrm{pg} / \mathrm{mL})$ & $264.7(85.1,684.4)$ & $127.2(72.5,788.2)$ & .96 & $483.5(105.5,748.1)$ & $90.2(28.1,221.9)$ & .12 \\
PDGF AA $(\mathrm{pg} / \mathrm{mL})$ & $9.5(5.1,26.2)$ & $14.4(3.5,35.0)$ & .76 & $11.9(8.0,31.0)$ & $4.9(2.8,8.7)$ & .06 \\
CRP $(\mathrm{mg} / \mathrm{L})$ & $9.0(4.0,21.5)$ & $5.5(3.0,15.2)$ & .47 & $8.0(4.0,19.0)$ & $7.0(3.5,20.2)$ & .79 \\
\hline
\end{tabular}

Values are median (interquartile range; 25th and 75th percentiles), unless otherwise indicated. The levels of biomarkers in the CF-LVAD recipient group; comparison between patients with no general bleeding versus patients with general bleeding events, and between patients with no GI bleeding versus patients with GI bleeding events. $G I$, Gastrointestinal; $B N P$, B-type natriuretic peptide; $C G M P$, cyclic guanosine monophosphate; $N O$, nitric oxide; $P D G F$, platelet-derived growth factor; $C R P$, C-reactive protein.

dehydrogenase nor white blood cell levels were associated with either GI or general bleeding events. No significant differences were found in either GI or general bleeding events between patients with ischemic versus nonischemic etiology, or for patients with an open versus closed aortic valve. Cox regression revealed that the probability of CF-LVAD recipients having GI bleeding is increased by [hazard ratio $\operatorname{Exp}(\mathrm{B})] 1.30(95 \%$ confidence interval: 1.00-1.88) for every unit of increase in cGMP levels $(P<.05)$.

\section{DISCUSSION}

Surgical implantation of a CF-LVAD is one of the most promising therapies available for patients who have heart failure. Nevertheless, the long-term effects of CF-LVAD support are not completely understood. We found that cGMP levels were increased significantly in CF-LVAD recipients and are associated with the risk of GI bleeding, but not with bleeding in general. This increase in cGMP may be related to a higher incidence of GI bleeding in CF-LVAD recipients, through impaired platelet aggregation. Although we did not specifically examine whether elevated cGMP levels in CF-LVAD recipients impair platelet aggregation, we suggest it as an area for future study.
The mechanisms by which cGMP inhibits platelet activation can be explained in terms of several pathways. ${ }^{12}$ One is that cGMP decreases the intercellular calcium concentration, resulting in low calcium availability for use in platelet activation and aggregation. In addition, the expression of glycoprotein IIb/IIIa on the platelet surface is decreased by cGMP, which leads to less crosslinking between fibrinogen and glycoprotein IIb/IIa integrin receptors, decreasing platelet-to-platelet interaction. Expression of both thromboxane $A_{2}$ receptor, which contributes to platelet aggregation, and P-selectin, an adhesion molecule on the platelet and endothelial surface, is inhibited by cGMP.

In our study, we found that CF-LVAD recipients have increased levels of cGMP, suggesting that the mechanisms through which platelets maintain the balance between clot formation and bleeding are disrupted. High cGMP levels in CF-LVAD recipients are noteworthy, especially given that CF-LVAD recipients have elevated levels of CRP, which interfere with the NO-cGMP pathway. ${ }^{18}$ We found no differences in the levels of NO between the CF-LVAD recipients and the healthy participants. Given that NO is a short-lived radical that readily reacts with small scavenger molecules, its detection is very difficult. ${ }^{19}$ A multicenter study using a larger group of CF-LVAD recipients may be required to determine the levels of $\mathrm{NO}$ in this patient population.
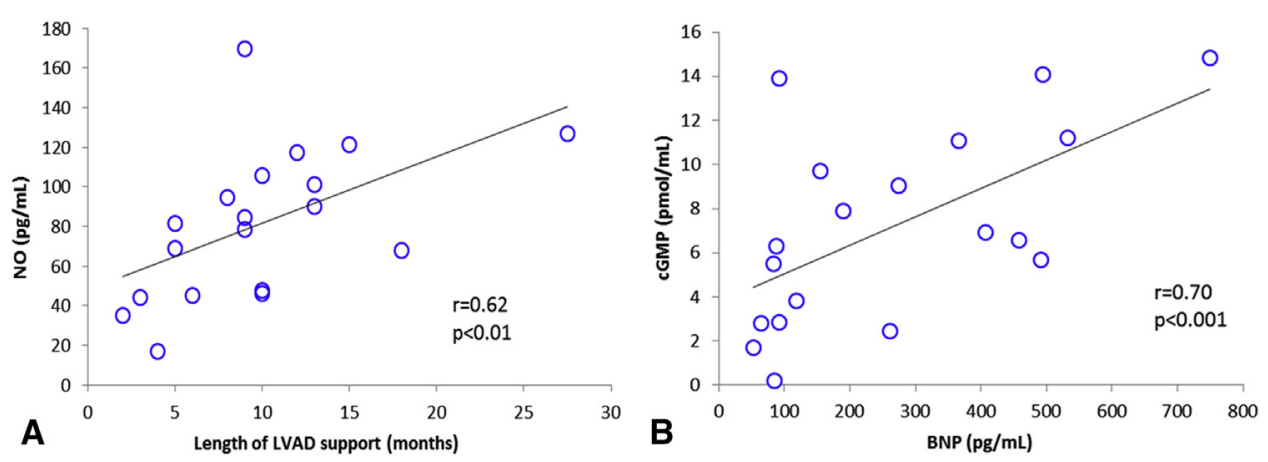

FIGURE 2. A, The relationship between cGMP (pmol/mL) and BNP (pg/mL) levels in CF-LVAD recipients (B) The relationship between NO (pmol/mL) and length of continuous LVAD support. NO, Nitric oxide; $L V A D$, left-ventricular assist device; $c G M P$, cyclic guanosine monophosphate; BNP, B-type natriuretic peptide. 
In addition to changes in hemostatic mechanisms, studies have found that after CF-LVAD implantation, acquired von Willebrand syndrome, which is characterized by platelet dysfunction, contributes to the high prevalence of bleeding. ${ }^{20,21}$ A landmark study ${ }^{5}$ reported that the most common bleeding in heart failure patients supported by a CF-LVAD is GI bleeding, and all patients had either a decreased or an absence of high molecular weight von Willebrand factor, which is required for platelet adhesion. The investigators suggested that these patients had acquired von Willebrand syndrome. They found that after heart transplantation and the removal of the CF-LVAD, which consequently restored pulsatile flow, the levels of high-molecular weight von Willebrand factor were normalized. This result suggests that the device was the cause of the bleeding diathesis and the resultant GI bleeding.

In our study, despite the abnormally higher cGMP levels in CF-LVAD recipients, compared with healthy controls, and even higher levels in patients who had heart failure, only the CF-LVAD recipients had GI bleeding and general bleeding events. One possibility is that several contributing factors acted in concert, including acquired von Willebrand factor syndrome and cGMP inhibition of platelet activation, resulting in this common morbidity in CF-LVAD recipients. Thus, the cGMP pathway may not be solely responsible for the increase in GI bleeding. This increase may be explained by the synergistic effects of high cGMP levels, acquired von Willebrand syndrome, low pulsatility, and anticoagulation therapy, which may predispose CF-LVAD recipients to a high risk of GI bleeding. A thorough investigation that includes all these factors will provide a more complete explanation of the causes of high rates of GI bleeding events in this patient population.

The HeartMate II is an axial flow pump, whereas the DuraHeart (Texas Heart Institute, Houston, Tex) and the HeartWare are centrifugal devices. In our study, all patients with GI bleeding $(21 \%)$ were supported with the HeartWare, whereas general bleeding events occurred in $10.5 \%$ of the patients supported with the HeartMate II, and in $21 \%$ with the HeartWare. Similar results were observed by Lalonde and colleagues ${ }^{22}$ in a retrospective study at our institution. The HeartWare devices were associated with a significantly higher incidence of GI bleeding, compared with the HeartMate II (31\% vs $0 \%$ of patients; $P<.01)$. Previously, studies showed that $22 \%$ of HeartMate II, ${ }^{23,24}$ and $13 \%$ to $15 \%$ of HeartWare ${ }^{25,26}$ recipients had GI bleeding events. In contrast, Meyer and colleagues $^{27}$ demonstrated similar reductions in highmolecular weight von Willebrand factor in recipients of centrifugal HeartWare, and those who had the axial flow HeartMate II. The effects of the DuraHeart centrifugal pump on hemocompatibility have not been well studied to date, and effects on hematologic parameters are therefore not well understood.

To prevent thrombosis, some CF-LVADs, such as the HeartMate II and the HeartWare, are designed with sintered titanium lining the inner surface of the devices. However, the effect of sintered devices on GI bleeding is not completely understood. In 2011, titanium sintering of the inflow cannula of HeartWare pumps was introduced, providing insight into the effect of a sintered titanium lining on hemocompatibility. ${ }^{28}$ In a retrospective analysis, thrombosis occurred in $4 \%$ of patients supported with the HeartWare with a sintered inflow cannula, versus $15 \%$ with a nonsintered pump, suggesting that a sintered titanium lining decreases thrombosis. However, the effect of a sintered lining on GI or general bleeding events in patients who have a left-ventricular assist device is not clear.

In the subgroup of patients supported with HeartMate II devices, for whom pulsatility index is available, pulsatility index was negatively correlated with cGMP levels. This finding provides further evidence that strategies to reduce cGMP level (such as lower pump speeds to achieve a higher pulsatility index) may reduce the risk of GI bleeding. Lower pump speeds often lead to more-frequent aortic valve opening, which may in itself reduce the risk of GI bleeding.

In addition, PDGF levels were lower in the CF-LVAD recipients and in the group with heart failure, compared with healthy participants. Our observations are in contrast to findings from in vivo ${ }^{14,15}$ and in vitro studies ${ }^{13,29}$ that demonstrated that contact of the blood with an artificial surface increases PDGF levels. For example, in cell culture models, exposure of platelets to a biventricular assist device surface induced the release of PDGF. ${ }^{13}$ Our findings that PDGF levels were lower in CF-LVAD recipients than in healthy participants suggests that, despite the contact of the blood with the artificial surface of the CF-LVAD, platelet activation was impaired, and consequently, systemic PDGF levels were low. We found that CRP levels were significantly higher in the CF-LVAD recipients compared with those in healthy participants. In addition, we found that despite the increase in the levels of CRP, which interfere with NO-cGMP production, ${ }^{18}$ cGMP levels were significantly higher in CF-LVAD recipients.

The source of elevated cGMP levels is not known. A well established finding is that excessive platelet activation is tightly regulated by platelets, via a paracrine mechanism. ${ }^{30}$ Platelet activation leads to the generation of NO by platelet endothelial NO synthase, which may either be released from the cell and interact with other platelets, or act as an intercellular signaling molecule. ${ }^{30}$ In our study, we observed significantly higher levels of cGMP, but we did not observe an increase in NO levels. A previous study demonstrated that cGMP efflux from activated platelets acts as a negative feedback regulator of platelet activation. ${ }^{31}$ 
A possible explanation is that blood contact with CF-LVAD activates platelets, resulting in increased platelet NO production, thereby inducing an elevation in cGMP production and subsequent cGMP release into the blood.

Alternatively, in CF-LVAD recipients, elevated cGMP level may be related to their heart failure. An additional pathway for the increase in cGMP in heart failure may be through the binding of atrial natriuretic peptide and BNP to natriuretic peptide receptor-A and activation of guanylate cyclase. ${ }^{32}$ However, in patients with severe heart failure, the natriuretic peptide system is attenuated, as a result of the downregulation of the natriuretic peptide receptor. ${ }^{33,34}$ In our study, BNP levels were significantly correlated with cGMP levels in the CF-LVAD recipients, but not in the other groups. However, BNP did not significantly predict GI bleeding events, which may be due to a large variability in BNP data in the CF-LVAD recipients. Larger, multicenter studies should examine whether BNP can predict GI bleeding events.

Several studies have found that in heart failure, cardiac myocytes and circulating monocytes express high levels of the NO-producing enzyme, inducible NO synthase, ${ }^{35-38}$ the production of which is triggered by inflammatory cytokines. $^{39}$ At low concentrations, NO stimulates guanylate cyclase activity and triggers the formation of cGMP. $^{39}$ The CF-LVAD support may not reverse some of the pathologic effects of the heart failure, and therefore, the high cGMP levels may persist from the heart failure from before CF-LVAD implantation and increase the risk of bleeding in patients with high cGMP levels. Future studies are required to identify the source of cGMP.

The main limitation of the study is that it is a singlecenter study, comparing small groups of patients with 3 different CF-LVAD types, to both patients who have heart failure and healthy participants. This investigation does not permit an in-depth study of the time course for the rise in cGMP levels, and the decrease in PDGF after CF-LVAD implantation. Further research with a larger cohort of CF-LVAD recipients, investigating patients with versus without bleeding events, is required to determine whether cGMP can be used as a clinical marker for predicting GI bleeding in this patient population. Nevertheless, despite our small sample size, we were able to demonstrate a significant correlation between high cGMP levels and GI bleeding in recipients of a CF-LVAD.

A longitudinal assessment of the variability of cGMP levels over time and the coincidence of GI bleeding should be made as well. In this study, with the exception of sildenafil, the medications used did not have an effect on cGMP levels. Additionally, the bleeding tendency in CF-LVAD recipients exceeds the normal levels that were observed in patients receiving anticoagulation treatment. ${ }^{40}$ However, we cannot exclude the possibility that anticoagulation use may increase cGMP levels in CFLVAD recipients, predisposing them to a higher risk of GI bleeding. Large, multicenter studies should investigate the effects of anticoagulation on cGMP levels as well as on GI bleeding events.

In conclusion, we found that GI bleeding in CF-LVAD recipients was associated with elevated cGMP levels. This novel finding may have clinical implications, as cGMP levels may be used as a clinical marker for increased risk of GI bleeding in CF-LVAD recipients. The cGMP inhibitory pathways may be contributing mechanisms for platelet impairment. Further studies are required to investigate the mechanisms underlying platelet dysfunction and GI bleeding in CF-LVAD recipients, and to determine potential new therapies.

\section{Conflict of Interest Statement}

Vivek Rao, MD, is a consultant to Terumo Corp (Tokyo), Thoratec Corp (Pleasanton, Calif), and HeartWare International, Inc (Framingham, Mass). All other authors have nothing to disclose with regard to commercial support.

\section{References}

1. Slaughter MS, Rogers JG, Milano CA, Russell SD, Conte JV, Feldman D, et al; HeartMate II Investigators. Advanced heart failure treated with continuous-flow left ventricular assist device. N Engl J Med. 2009;361:2241-51.

2. John R, Kamdar F, Liao K, Colvin-Adams M, Miller L, Joyce L, et al. Low thromboembolic risk for patients with the Heartmate II left ventricular assist device. J Thorac Cardiovasc Surg. 2008;136:1318-23.

3. Kirklin JK, Naftel DC, Kormos RL, Stevenson LW, Pagani FD, Miller MA, et al. Third INTERMACS Annual Report: the evolution of destination therapy in the United States. J Heart Lung Transplant. 2011;30:115-23.

4. Demirozu ZT, Radovancevic R, Hochman LF, Gregoric ID, Letsou GV, Kar B, et al. Arteriovenous malformation and gastrointestinal bleeding in patients with the HeartMate II left ventricular assist device. J Heart Lung Transplant. 2011;30:849-53.

5. Uriel N, Pak SW, Jorde UP, Jude B, Susen S, Vincentelli A, et al. Acquired von Willebrand syndrome after continuous-flow mechanical device support contributes to a high prevalence of bleeding during long-term support and at the time of transplantation. J Am Coll Cardiol. 2010;56:1207-13.

6. Crow S, John R, Boyle A, Shumway S, Liao K, Colvin-Adams M, et al. Gastrointestinal bleeding rates in recipients of nonpulsatile and pulsatile left ventricular assist devices. J Thorac Cardiovasc Surg. 2009;137:208-15.

7. Fiaccadori E, Maggiore U, Clima B, Melfa L, Rotelli C, Borghetti A. Incidence, risk factors, and prognosis of gastrointestinal hemorrhage complicating acute renal failure. Kidney Int. 2001;59:1510-9.

8. Wasse H, Gillen DL, Ball AM, Kestenbaum BR, Seliger SL, Sherrard D, et al. Risk factors for upper gastrointestinal bleeding among end-stage renal disease patients. Kidney Int. 2003;64:1455-61.

9. Hedges SJ, Dehoney SB, Hooper JS, Amanzadeh J, Busti AJ. Evidence-based treatment recommendations for uremic bleeding. Nat Clin Pract Nephrol. 2007;3:138-53.

10. Noris M, Benigni A, Boccardo P, Aiello S, Gaspari F, Todeschini M, et al. Enhanced nitric oxide synthesis in uremia: implications for platelet dysfunction and dialysis hypotension. Kidney Int. 1993;44:445-50.

11. Remuzzi G, Perico N, Zoja C, Corna D, Macconi D, Vigano G. Role of endothelium-derived nitric oxide in the bleeding tendency of uremia. J Clin Invest. 1990;86:1768-71.

12. Jin RC, Loscalzo J. Vascular nitric oxide: formation and function. J Blood Med. 2010;2010:147-62.

13. Hasper D, Hummel M, Hetzer R, Volk HD. Blood contact with artificial surfaces during BVAD support. Int J Artif Organs. 1996;19:590-6.

14. Cianciolo G, Stefoni S, Donati G, De Pascalis A, Iannelli S, Manna C, et al. Intra- and post-dialytic platelet activation and PDGF-AB release: cellulose 
diacetate vs polysulfone membranes. Nephrol Dial Transplant. 2001;16: 1222-9.

15. Cianciolo G, Stefoni S, Zanchelli F, Iannelli S, Coli L, Borgnino LC, et al. PDGF$\mathrm{AB}$ release during and after haemodialysis procedure. Nephrol Dial Transplant. 1999;14:2413-9.

16. Caruso R, Trunfio S, Milazzo F, Campolo J, De Maria R, Colombo T, et al. Early expression of pro- and anti-inflammatory cytokines in left ventricular assist device recipients with multiple organ failure syndrome. ASAIO J. 2010; $56: 313-8$.

17. Loebe M, Koster A, Sanger S, Potapov EV, Kuppe H, Noon GP, et al. Inflammatory response after implantation of a left ventricular assist device: comparison between the axial flow MicroMed DeBakey VAD and the pulsatile Novacor device. ASAIO J. 2001;47:272-4.

18. Verma S, Szmitko PE, Ridker PM. C-reactive protein comes of age. Nat Clin Pract Cardiovasc Med. 2005;2:29-36. quiz 58.

19. Moshage H. Simple and reliable measurement of nitric oxide metabolites in plasma. Clin Chem. 2009;55:1881-2.

20. Heilmann C, Geisen U, Beyersdorf F, Nakamura L, Trummer G, BerchtoldHerz M, et al. Acquired Von Willebrand syndrome is an early-onset problem in ventricular assist device patients. Eur J Cardiothorac Surg. 2011;40: 1328-33; discussion 1233.

21. Geisen U, Heilmann C, Beyersdorf F, Benk C, Berchtold-Herz M, Schlensak C, et al. Non-surgical bleeding in patients with ventricular assist devices could be explained by acquired von Willebrand disease. Eur J Cardiothorac Surg. 2008; 33:679-84.

22. Lalonde SD, Alba AC, Rigobon A, Ross HJ, Delgado DH, Billia F, et al. Clinical differences between continuous flow ventricular assist devices: a comparison between HeartMate II and HeartWare HVAD. J Card Surg. 2013; 28:604-10.

23. Morgan JA, Paone G, Nemeh HW, Henry SE, Patel R, Vavra J, et al. Gastrointestinal bleeding with the HeartMate II left ventricular assist device. $J$ Heart Lung Transplant. 2012;31:715-8.

24. Harvey L, Holley CT, John R. Gastrointestinal bleed after left ventricular assist device implantation: incidence, management, and prevention. Ann Cardiothorac Surg. 2014;3:475-9.

25. Goldstein DJ, Aaronson KD, Tatooles AJ, Silvestry SC, Jeevanandam V, Gordon R, et al; ADVANCE Trial Investigators. Gastrointestinal bleeding in recipients of the HeartWare ventricular assist system. JACC Heart Fail. 2015; 3:303-13.

26. Slaughter MS, Pagani FD, McGee EC, Birks EJ, Cotts WG, Gregoric I, et al. HeartWare Bridge to Transplant ADVANCE Trial Investigators. HeartWare ventricular assist system for bridge to transplant: combined results of the bridge to transplant and continued access protocol trial. J Heart Lung Transplant. 2013; 32:675-83.

27. Meyer AL, Malehsa D, Budde U, Bara C, Haverich A, Strueber M. Acquired von Willebrand syndrome in patients with a centrifugal or axial continuous flow left ventricular assist device. JACC Heart Fail. 2014;2:141-5.
28. Soltani S, Kaufmann F, Vierecke J, Kretzschmar A, Hennig E, Stein J, et al. Design changes in continuous-flow left ventricular assist devices and life-threatening pump malfunctions. Eur J Cardiothorac Surg. 2015; 47:984-9.

29. Lappegard KT, Bergseth G, Riesenfeld J, Pharo A, Magotti P, Lambris JD, et al. The artificial surface-induced whole blood inflammatory reaction revealed by increases in a series of chemokines and growth factors is largely complement dependent. J Biomed Mater Res A. 2008;87:129-35.

30. Naseem KM, Riba R. Unresolved roles of platelet nitric oxide synthase. J Thromb Haemost. 2008;6:10-9.

31. Wu XB, Brune B, Von Appen F, Ullrich V. Efflux of cyclic GMP from activated human platelets. Mol Pharmacol. 1993;43:564-8.

32. Nishikimi T, Maeda N, Matsuoka $H$. The role of natriuretic peptides in cardioprotection. Cardiovasc Res. 2006;69:318-28.

33. Tsutamoto T, Wada A, Maeda K, Hisanaga T, Maeda Y, Fukai D, et al Attenuation of compensation of endogenous cardiac natriuretic peptide system in chronic heart failure: prognostic role of plasma brain natriuretic peptide concentration in patients with chronic symptomatic left ventricular dysfunction. Circulation. 1997;96:509-16.

34. Tsutamoto T, Kanamori T, Morigami N, Sugimoto Y, Yamaoka O, Kinoshita M. Possibility of downregulation of atrial natriuretic peptide receptor coupled to guanylate cyclase in peripheral vascular beds of patients with chronic severe heart failure. Circulation. 1993;87:70-5.

35. Haywood GA, Tsao PS, von der Leyen HE, Mann MJ, Keeling PJ, Trindade PT, et al. Expression of inducible nitric oxide synthase in human heart failure. Circulation. 1996;93:1087-94.

36. Comini L, Bachetti T, Agnoletti L, Gaia G, Curello S, Milanesi B, et al. Induction of functional inducible nitric oxide synthase in monocytes of patients with congestive heart failure. Link with tumour necrosis factor-alpha. Eur Heart J. 1999;20:1503-13.

37. Orus J, Heras M, Morales-Ruiz M, Leivas A, Roig E, Rigol M, et al Nitric oxide synthase II mRNA expression in cardiac tissue of patients with heart failure undergoing cardiac transplantation. J Heart Lung Transplant. 2000;19: $139-44$.

38. Ferreiro CR, Chagas AC, Carvalho MH, Dantas AP, Scavone C, Souza LC, et al. Expression of inducible nitric oxide synthase is increased in patients with heart failure due to ischemic disease. Braz J Med Biol Res. 2004;37: 1313-20.

39. Beck KF, Eberhardt W, Frank S, Huwiler A, Messmer UK, Muhl H, et al Inducible NO synthase: role in cellular signalling. J Exp Biol. 1999;202: 645-53.

40. Goldstein DJ, Beauford RB. Left ventricular assist devices and bleeding: adding insult to injury. Ann Thorac Surg. 2003;75(6 Suppl):S42-7.

Key Words: continuous-flow left ventricular assist device, gastrointestinal bleeding, cyclic guanosine monophosphate 